\title{
Surveillance of Anthrax Foci Across Pipeline Constructions in Georgia, 2003-2014
}

\author{
Nikoloz Tsertsvadze, Lile Malania, Nato Abazashvili, Julieta Manvelian, Mariam \\ Broladze and Paata Imnadze
}

National Center for Disease Control and Public Health of Georgia, Tbilisi, Georgia

\section{Introduction}

Anthrax is a widely distributed endemic infection in Georgia, affecting nearly the entire country. Many of the human cases that are annually registered are agriculturally acquired. Anthrax remains a public health risk due to active, resistant soil foci. More than 2,000 anthrax affected areas are registered in the country; around $10 \%$ of them are active. Recent reports have indicated an increase in the number of human cases as a result of contact with the environment, this is hypothesized to be due to expansion of affected foci, and this has raised concerns of the disease spreading to new areas.

The control of anthrax foci is one of the main goals of the public health and veterinary service's in Georgia. A surveillance program of anthrax foci across pipeline constructions in Georgia has been ongoing since 2003. Field trips are conducted by National Center for Disease Control and Public Health mobile teams to investigate each possible affected area across pipeline constructions.

\section{Methods}

During 2003-2014, 9,296 soil samples were collected from 19 different regions of the country and screened for the presence of Bacillus anthracis using bacteriological and molecular methods. Laboratory investigations at NCDC were performed using Threat Agent Detection and Response (TADR) algorithms.

\section{Results}

The data shows that areas around several construction sites were contaminated, therefore, workers had a potential exposure risk. Overall, 40 isolates of $B$. anthracis were obtained from the soil samples. Phenotypic (DTRA approved algorithm) and genetic (e.g., SNP, MLVA) profile studies were conducted for strain characterization.

\section{Conclusions}

Affected territories were isolated and decontaminated, and material that provided education on how to prevent the spread of anthrax in construction zones was provided to appropriate populations. The data obtained from this study highlights the importance of surveillance programs on especially dangerous pathogens.

\section{Keywords}

Bacillus anthracis; anthrax; surveillance; pipeline; decontamination 